\title{
Linewidth Study of 40-GHz Passively Mode-Locked Multimode Semiconductor Lasers
}

\author{
Ramón Maldonado-Basilio, Sylwester Latkovsky, Frederic Surre, and Pascal Landais \\ School of Electronic Engineering, Dublin City University, Glasnevin, Dublin 9, Ireland
}

Passively mode-locked (PML) lasers have recently been investigated for pulse signal generation as they exhibit a periodic time variation of their output emission under continuous wave bias conditions. These devices allow a cost effective solution for pulse waveform signals generation at $40 \mathrm{GHz}$ as they do not require any direct or external modulation. There are several types of configurations for edge emitting PML lasers. Among the most important are those based on gain-coupled distributed feedback (DFB) lasers [1], distributed Bragg reflector (DBR) lasers [2] and Fabry-Pérot (FP) cavities [3].

In some of the mentioned works, attention has been focused either on the self-pulsating (SP) frequency evolution in terms of the bias current or on the radio-frequency (RF) signal linewidth, considering a fixed number of longitudinal modes selected from the optical emission spectrum. In this work, an experimental study of the impact of the number of longitudinal modes on the linewidth of $\sim 40 \mathrm{GHz}$ signals generated by various passively mode-locked multimode semiconductor lasers is addressed. Four of these devices under test (DUT) are multi-quantum wells InAlGaAs FP lasers with a free spectral range at $40 \mathrm{GHz}$, whose structure is similar to that of the devices reported in [4]. They have a one-dimensional photonic band-gap embedded in their structure, which is achieved by etching shallow grooves on the p-side of the laser junction. They have been engineered to exhibit a specific number of longitudinal modes: $4,5,10$, and 22 modes, respectively [5]. These four DUTs have a threshold current around $27 \mathrm{~mA}$ and a maximum average collected power around $0.5 \mathrm{~mW}$ when operating at $100 \mathrm{~mA}$. Another DUT is a bulk DBR laser exhibiting 3 lasing modes and its main features are described in [6]. The last device is a quantum-dash FP laser characterized by 40 lasing modes. It is important to stress that we analyze the total number of modes oscillating into the laser cavity, without implementing an external optical processing such as a filtering out some longitudinal modes at the laser output, as it has been studied in [3,7].

In our experiment, the output of each DUT is coupled into a single mode fiber (SMF) and measured both electrically and optically. As it is illustrated in Fig. 1, our experimental results show that, independently from the nature of the cavity and the active media, the FWHM-linewidth of the $\sim 40 \mathrm{GHz}$ signal generated by these lasers is reduced as the number of longitudinal modes selected by the laser cavity is increased. A criterion to identify the passively mode-locking mechanism in these devices is given by $\Delta v_{R F}<<\sum \Delta v_{i}$, where $\Delta v_{R F}$ is the FWHMlinewidth of the RF signal and $\Delta v_{i}$ is the linewidth of the $\mathrm{i}$-th optical mode. The ratio between these two variables is illustrated in Fig. 2 for each DUT in terms of the number of longitudinal modes oscillating into the laser cavity.

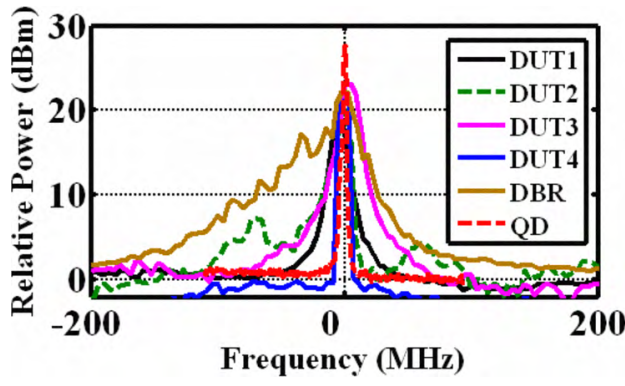

Fig. 1. RF-signal generated by the analyzed DUTs

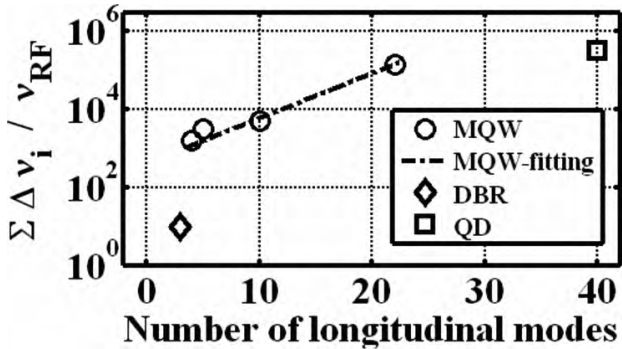

Fig. 2. Ratio $\Sigma \Delta v_{i} / \Delta v_{R F}$ in terms of the longitudinal modes oscillating in the laser cavity

\section{References}

[1] M. Möhrle, B. Sartorius, C. Bornholdt, S. Bauer, O. Brox, A. Sigmund, R. Steingrüber, M. Radziunas and H.-J. Wünsche. "Detuned grating multisection-RW-DFB lasers for high-speed optical signal processing". J. Sel. Topics Quantum Electron. 7 (2), 217-223 (2001). [2] J. Renaudier, G. H. Duan, P. Landais, and P. Gallion. "Phase correlation and linewidth reduction of $40 \mathrm{GHz}$ self-pulsation in distributed Bragg reflector semiconductor laser”. J. Quantum Electron. 43(2), 147-156 (2007).

[3] Kenji Sato. "Optical pulse generation using Fabry-Pérot lasers under continuous-wave operation". J. Sel. Topics Quantum Electron. 9(5), 1288-1293 (2003).

[4] Sylwester Latkowsky, Frederic Surre, Ramon Maldonado-Basilio, and Pascal Landais. "Investigation on the origin of terahertz waves generated by dc-biased multimode semiconductor lasers at room temperature". Appl. Phys. Lett. 93(24), 241110 (2008).

[5] Pascal Landais, Patent No. S2005/0251. Ireland (26 April 2005).

[6] A. Shen, J-G. Provost, A. Akrout, B. Rousseau, F. Lelarge, O. Legouezigou, F. Pommereau, F. Poingt, L. Legouezigou, G-H. Duan, and A. Ramdane. "Low confinement factor quantum dash (QD) mode-locked Fabry-Perot (FP) laser diode for tunable pulse generation", in OFC'08, OThK1 (2008).

[7] S. Latkowski, F. Surre, P. Landais and G.-H. Duan. "All-optical syncronization of a $40 \mathrm{GHz}$ self-pulsating distributed Bragg reflector laser to return-to-zero 10, 20, and $40 \mathrm{Gbits} / \mathrm{s}$ data stream". Optics Communications (to be published). 